\title{
126. NUEVAS LOCALIDADES DE ESPECIES INTERESANTES EN DOÑANA Y LA COSTA DE HUELVA (SW ESPAÑA)
}

\author{
Itziar LÓPEZ-ALBACETE, María de los Ángeles DE LAS HERAS, Enrique SÁNCHEZ- \\ GULLÓN, Pedro WEICKERT, Pablo J. HIDALGO y Adolfo Francisco MUÑOZ
}

Recibido el 23 de febrero de 2007, aceptado para su publicación el 10 de junio de 2007

Publicado "on line" en junio de 2007

New floristic records of species for Doñana and Huelva (SW Spain)

Palabras clave. Flora, Doñana, Calystegia soldanella, Herniaria cinerea, Trigonella monspeliaca, Viola lactea, Wolffia arrhiza, especies amenazadas.

Key words. Flora, Doñana, Calystegia soldanella, Herniaria cinerea, Trigonella monspeliaca, Viola lactea, Wolffia arrhiza, threatened species.

Las localidades mencionadas en el presente trabajo han surgido de un estudio que están realizando algunos de los autores sobre la vegetación de los Parques Nacional y Natural de Doñana, integrado en el proyecto "Cartografía y Evaluación de la vegetación y flora a escala de detalle de los ecosistemas forestales de las provincias de Cádiz y Huelva" financiado por la Consejería de Medio Ambiente de la Junta de Andalucía. No obstante, dado que algunas de las especies incluidas han sido también localizadas en otros puntos de la costa de Huelva, fuera del ámbito de dichos parques, se ha considerado conveniente incluirlos en la misma publicación de forma que complementen los conocimientos sobre la distribución de estos taxones.

Calystegia soldanella (L.) R. Br., Prodr. Fl. Nov. Holl. 484 (1810)

HUELVA. Almonte: Parque Nacional de Doñana, tren de dunas móviles, en dunas embrionarias cerca de Torre Carboneras. UTM: 29S723871/4092271, 24-V-06. Leg: I. López \& A. F. Muñoz. COFC 30383. Ayamonte: Punta del Moral. En sistema dunar de Ammophiletea. UTM: 29SPB4717, 6-II-2007. Leg.: E. Sánchez Gullón
\& P. Weickert. COFC 30382.

Este taxón tiene una distribución subcosmopolita, presentándose en arenales costeros de casi todo el Globo. En Europa se encuentra desde Escocia hasta Dinamarca, así como en toda la costa sur y oeste (Brummitt, 1996). En España está muy bien representado en toda la Cordillera Cantábrica y en la costa este mediterránea, sin embargo es muy escaso en el suroeste peninsular.

En Andalucía la presencia de este taxón fue registrada por primera vez en la provincia de Cádiz por Pérez Lara en 1889, en Algeciras y Palmones. Valdés (1987: 369), cita en Cádiz esta convolvulácea, muy localizada en la comarca de Algeciras, donde aparece en sistemas dunares de arenas originadas con materiales sedimentarios de calcarenitas.

En el año 2002 se cita por primera vez en la provincia de Huelva, en las proximidades de la laguna del Portil (Punta Umbría) ampliando así su distribución al Litoral Onubense (Sánchez Gullón \& Rubio García, 2002). A partir de entonces, en el informe sobre la biodiversidad de Doñana (García-Novo \& Marín-Cabrera, 2005) se recoge en el listado de especies vegetales existentes dentro del Parque Nacional de Doñana, sin especificar localidad ni aportar pliego testigo que avale su presencia en este territorio, y Gallego Fernández, et al. (2006), lo citan en el litoral 
occidental onubense, concretamente en el Paraje Natural Marismas del Río Piedras y en la Flecha del Rompido.

La especie se recolectó en comunidades de Ammophiletea Br.Bl. \& Tüxen tanto en el tren de dunas móviles del Parque Nacional como en la Punta del Moral, siendo por tanto las localidades más orientales y occidentales, respectivamente, de las citas dadas para la provincia. Dado que esta especie se ha incluido con la categoría de vulnerable en la lista roja de la flora vascular andaluza (Cabezudo y Talavera, 2005), parece interesante la aportación de nuevas poblaciones, más aún dentro de un espacio protegido tan emblemático como es el Parque Nacional de Doñana.

Herniaria cinerea DC. in Lam. \& DC., Fl. Franç., ed. 3, 5: 375 (1805)

HUELVA: Almonte: Reserva Biológica de Doñana (Camino de Palacio). UTM: 29S 726233/ 4096989, 3-V-2006. Leg: P. Hidalgo, I. López \& A. F. Muñoz. COFC 30381.

Taxón cuya distribución mundial abarca el sur de Europa, norte de África y oeste de Asia. En España según Chaudhri (1990) se encuentra dispersa por toda la Península y las Islas Baleares, en concreto en Andalucía la incluye en todas las provincias.

En Huelva la cita más antigua de esta especie se encuentra en el trabajo de Vicioso (1948) donde se registra la presencia en dos localidades: Paymogo, en el oeste del Andévalo, y Cumbres Mayores, en el norte, cerca de la provincia de Badajoz; aunque posteriormente Devesa (1987) sólo la incluye en los sectores de la Campiña Alta y Condado-Aljarafe.

En el informe sobre la biodiversidad de Doñana (García-Novo \& Marín-Cabrera, 2005) aparece en el listado de especies presentes en el Parque Nacional, sin embargo al igual que ocurre con el taxón anterior, no se especifica localidad ni se aporta pliego alguno, por lo que en el presente trabajo se constata la presencia de este taxón en dicho parque y en el litoral onubense.

Se recolectó en la Reserva Biológica de Doñana, en las cunetas del camino de entrada al Palacio, asociada a otras especies ruderales cuya presencia dentro de Doñana está relegada a la existencia de infraestructuras viarias y edificaciones.

Trigonella monspeliaca L., Sp. Pl. 777 (1753)

HUELVA. Almonte: Reserva Biológica de Doñana (Camino de Palacio). UTM: 29S 726233/ 4096958, 3-V-2006. Leg: P. Hidalgo, I. López \& A. F. Muñoz. COFC 30380

Taxón de distribución amplia, se encuentra en el centro y sur de Europa, norte de África y suroeste de Asia hasta el norte de Irán. En la Península y Baleares está también ampliamente distribuido (Hedge y Sales, 2000), habiendo sido citado en Andalucía en todas las provincias. La provincia de Huelva no figuraba en el área de distribución dada para esta especie por Domínguez (1987), siendo indicada por primera vez por Sánchez Gullón \& Rubio García (1999) en la localidad de Alpizar (Paterna del Campo).

La población que se aporta en este trabajo se recolectó en la Reserva Biológica de Doñana, en el camino de entrada hacia el Palacio, por lo que su presencia, como la de otros muchos taxones, está ligada a la presencia de suelos alóctonos para la construcción de infraestructuras viarias. Con esta nueva localidad se amplia la distribución del taxón al litoral onubense.

Viola lactea Sm., Engl. Bot. 7, tab. 445 (1797)

HUELVA. Almonte: Parque Natural de Doñana, Ribetehilos: Laguna del Galápago. UTM: 29SQB08651221, 31-V-06. Leg.: I. López \& A. F. Muñoz. COFC 30379.

Esta especie se distribuye por las comarcas atlánticas europeas, desde las Islas Británicas hasta Portugal (Fernández Casado y Nava Fernández, 1987). En la Península Ibérica se presenta a lo largo de toda la cornisa Cantábrica, Galicia y el litoral portugués. Según Lainz y Muñoz Garmendia (1992) y Muñoz Garmendia et al. (1993) señalaron su aparición en la provincia de Huelva, a partir de plantas recolectadas por S. Talavera en el Arroyo de la Rocina (Almonte). A esta localidad debe añadirse una cita de la Laguna de las Madres (Moguer), proveniente del herbario de B. Weickert, herborizada en 1956, antes de la extracción de turba en dicha laguna (Sánchez Gullón y Weickert, 2002), que parece ser es la población mas meridional conocida de esta especie.

La nueva localidad que se aporta se 
encuentra en el Parque Natural de Doñana, más concretamente en la zona de turberas conocida como Ribetehilos. La especie se recolectó en el vaso de una de las turberas, donde predominan las comunidades de herbáceas perennes pertenecientes al orden Molinio-Holoschoenion vulgaris Br.-Bl., incluidas como áreas de especial protección para su conservación en el Anexo I de la Directiva Hábitats (Directiva 92/43/CEE de 21 de mayo de 1992). Estos pastizales están dominados por Molinia caerulea (L.) Moench y Schoenus nigricans L., y se desarrollan entre los típicos brezales de turbera pertenecientes a la asociación Erico ciliaris-Ulicetum lusitanici Rivas-Martínez, Costa, Castroviejo \& E. Valdés. La importancia de esta cita radica en la reciente catalogación de esta especie en la Lista Roja de la flora vascular de Andalucía como especie en peligro crítico basándose en una única población conocida de la especie en esta región (Cabezudo y Talavera, 2005).

Wolffia arrhiza (L.) Horkel ex Wimm., Fl. Schles. ed. 3: 140 (1857)

HUELVA. Almonte: Parque Natural de Doñana, Lagunas de Castilla, Zacallón de los Americanos, UTM: 29SQB10940712, 12-IX-06. Leg.: C. Gutiérrez \& I. López. COFC 30378. HUELVA. Rociana del Condado: Arroyo del Avispero, UTM: 29SQB0726, 29-VI-2006. Leg: E. Sánchez Gullón \& P. Weickert. COFC 30377.

La distribución de esta especie incluye Eurasia y el Norte de África, habiendo sido indicada también para Oceanía. En España ha sido recolectada en La Mancha, Extremadura (Cirujano et al., 1992) y en Andalucía, comunidad esta última donde se ha citado en las provincias de Cádiz y Huelva (Talavera, 1987; García Murillo, 2000).

En Huelva García Murillo (2000) la ha recolectado en la laguna del Portil (Punta Umbría), la laguna de la Jara (Moguer) y en varias localidades del Parque Nacional de Doñana, aunque en el estudio de Galán de Mera y Castroviejo (2005), acerca del tratamiento de Lemnaceae en Flora Ibérica, sólo se ratifican dos localidades (Punta Umbría y Doñana: Laguna del Hondón). Fernández Zamudio et al. (2006) aporta una nueva localidad de $W$. arrhiza para el Parque Nacional de Doñana, en el Zacallón de Regianes.
Wolffia arrhiza está incluida en el catálogo de especies vulnerables de la'Ley nacional de la Flora y la Fauna Silvestres (Ley 8/2003 de 28 de octubre) así como en el Catálogo Andaluz de especies de la flora silvestre amenazada (Decreto 104/1994 de 10 de mayo). El Libro Rojo de la Flora Silvestre Amenazada de Andalucía, también considera esta especie como vulnerable (García Murillo, 2000), Cabezudo \& Talavera (2005) la incluyen entre la especies en Peligro. Todo ello pone de manifiesto la importancia del conocimiento de la distribución de esta especie y da valor a estas dos nuevas citas, teniendo además en cuenta, que se localiza en un área donde no había sido recolectada anteriormente, ya que entre las citas más occidentales (E1 Portil y Moguer) y las del Parque Nacional de Doñana, las nuevas localidades que aquí se dan a conocer se sitúan en el vasto territorio que abarca el Parque Natural de Doñana y en el término municipal de Rociana del Condado.

En el Parque Natural la especie se recolectó en un charcón que mantiene agua durante todo el año, donde se ha registrado la presencia de diversas especies de plantas acuáticas como Potamogeton lucens L., Myriophyllum alterniflorum DC., Ranunculus peltatus Schrank y Lemna gibba L. La población de Rociana se recolectó en un zacallón higroturboso del arroyo del Avispero, tributario del arroyo de la Rocina, acompañada por Lemna minor L. y Utricularia exoleta $\mathrm{R}$. Br.

\section{BIBLIOGRAFÍA}

BRUMMITT, R. K. -1996-'Calystegia en: Tutin'et al. (eds.) Flora Europaea Vol. III: 78-79. Cambridge University Press. Cambridge.

CABEZUDO, B. y S. TALAVERA (Coordinadores) -2005- Lista roja de la flora vascular de Andalucía. Junta de Andalucía. Sevilla.

CHAUDHRI, M. N. -1990- Herniaria. En: S. Castroviejo (coor.) Flora Iberica Vol. II: 118 134. Real Jardín Botánico, CSIC. Madrid.

CIRUJANO, S., M. VELAYOS, F. CASTILLA y M. GIL PINILLA -1992- Criterios para la valoración de las lagunas y humedales españoles (Península Ibérica e Islas Baleares). ICONA. Madrid. 
DEVESA, J. A. -1987- Herniaria. En: B. Valdés, S. Talavera y E. Fernández-Galiano (eds.) Flora de Andalucía Occidental Vol. I: 210212. Ketres Editora. Barcelona.

DOMÍNGUEZ, E. -1987- Trigonella. En: B. Valdés, S. Talavera y E. Fernández-Galiano (eds.) Flora de Andalucía Occidental Vol. II: 127-129. Ketres Editora. Barcelona.

FERNÁNDEZ CASADO, M. A. y H. S. NAVA FERNÁNDEZ -1987- Estudio de una violeta endémica de la Península Ibérica. Anales Jard. Bot. Madrid 44(2): 299-307.

FERNÁNDEZ ZAMUDIO, R., S. CIRUJANO, I. NIETO GIL, M. D. COBO, A. SOUSA MARTÍN y P. GARCÍA MURILLO -2006Novedades florísticas en el Parque Nacional de Doñana (SW España). Acta Bot. Malacitana 31: 191-195.

GALÁN DE MERA, A y S. CASTROVIEJO 2005- Acerca del tratamiento de'Lemnaceae en Flora Ibérica. Acta Bot. Malacitana 29: 149-150.

GALLEGO FERNÁNDEZ, J. B., S. MUÑOZ VALLÉS y C. DELLAFIORE -2006- Flora y vegetación de la flecha litoral de Nueva Umbría. Excmo. Ayuntamiento de Lepe. Lepe.

GARCÍA MURILLO, P. -2000- Wolffia arrhiza. En: G. Blanca et al. (eds.) Libro Rojo de la Flora Silvestre Amenazada de Andalucía Tomo II: Especies Vulnerables: 373-375. Junta de Andalucía. Sevilla.

GARCÍA-NOVO, F. y C. MARTÍN-CABRERA -2005- Doñana: Agua y Biosfera. Doñana 2005, Confederación hidrográfica del Guadalquivir, Ministerio de Medio Ambiente. Madrid.

HEDGE, I. C y F. SALES -2000- Trigonella. En : S. Castroviejo (coor.) Flora Iberica Vol. VII(II): 731-741. Real Jardín Botánico, CSIC. Madrid.

LAINZ, M. y F. MUÑOZ GARMENDIA -1992De re chorologica, nova et vetera. VII. Anales Jard. Bot. Madrid 50(2): 269.

MUÑOZ GARMENDIA, F., P. MONTSERRAT, M. LAIINZ y J. J. ALDASORO -1993-Viola. En: S. Castroviejo (coor.) Flora Ibérica Vol. III: 276-317. Real Jardín Botánico, CSIC. Madrid.

PÉREZ LARA, J. M. -1889- Florula gaditana.
Pars tertia. Anales Soc. Esp. Hist. Nat. 18(1): 35-143.

SÁNCHEZ GULLÓN, E. y J. C. RUBiO GARCÍA -1999- Novedades florísticas para el litoral de Huelva y provincia I. Lagascalia 21(1): 7-16.

SÁNCHEZ GULLÓN, E. y J. C. RUBIO GARCÍA -2002- Novedades florísticas para el litoral de Huelva y provincia II. Lagascalia 22: 7-19.

SÁNCHEZ GULLÓN E. y P. WEICKERT -2002Plantas del herbario de D. Bruno Weickert herborizadas en la provincia de Huelva entre los años 1950/1974. Lagascalia 22: 172-179.

TALAVERA, S. -1987- Wolffia. En: B. Valdés, S. Talavera y E. Fernández-Galiano (eds.) Flora de Andalucía Occidental Vol III: 211. Ketres Editora. Barcelona.

VALDÉS, B. -1987- Calystegia. En: B. Valdés, S. Talavera y E. Fernández-Galiano (eds.) Flora de Andalucía Occidental Vol. II: 369. Ketres Editora. Barcelona.

VICIOSO, C. -1948- Notas sobre la flora española. Anales Jard. Bot. Madrid 6(2): 592.

Dirección de los autores. I. López-Albacete, M. A. de las Heras, P. J. Hidalgo y A. F. Muñoz: Dpto. Biología Ambiental y Salud Pública. Facultad de Ciencias Experimentales. Universidad de Huelva-Campus el Carmen. Avda. Fuerzas Armadas s/n, 21071 Huelva; e-mail: ; E. Sánchez Gullón y P. Weickert: Paraje Natural Marismas del Odiel, Ctra del Dique Juan Carlos I, Apdo 720, E-21071. Huelva.

Autor para correspondencia: itziar.lopez@dbasp.uhu.es 